\title{
ON HASSE ZETA FUNCTIONS OF GROUP ALGEBRAS OF ALMOST NILPOTENT GROUPS
}

\author{
TAKAKo FuKaYA
}

\section{Introduction}

1.1. In the paper $[\mathrm{Fu}]$, we generalized the Hasse zeta functions $\zeta_{A}(s)$ of commutative finitely generated rings $A$ over the ring $Z$ of integers, to noncommutative rings (concerning the definition, see below), and computed the zeta functions of some rings. The examples suggested a strong relationship between the convergence of the Hasse zeta functions and the Gelfand-Kirillov dimensions of the rings (concerning the definition of the Gelfand-Kirillov dimension, see section 2). So in $[\mathrm{Fu}]$, we conjectured the relationship between them (see section 2 ). In the present paper, we consider this conjecture in the case of group rings.

For a (not necessarily commutative) finitely generated $\operatorname{ring} A$ over $Z$, in [Fu] we defined the Hasse zeta function $\zeta_{A}(s)$ of $A$ by

$$
\zeta_{A}(s)=\prod_{M}\left(1-N(M)^{-s}\right)^{-1}
$$

where $M$ runs over the isomorphism classes of finite simple $A$-modules and $N(M)=\sharp \operatorname{End}_{A}(M)$.

We say $\zeta_{A}(s)$ converges if there exists a real number $d$ such that the product defining $\zeta_{A}(s)$ absolutely converges when $\operatorname{Re}(s)>d$, and we say $\zeta_{A}(s)$ diverges otherwise. In fact, the function $\zeta_{A}(s)$ diverges for some rings $A$.

The purpose of this paper is to prove

THEOREM 1.2. Let $G$ be a finitely generated group which has a nilpotent subgroup of finite index, and let $R$ be a finitely generated commutative ring over $Z$. Let $A$ be the group ring $R[G]$. Then the function $\zeta_{A}(s)$ converges.

We call a group which has nilpotent subgroup of finite index, an almost nilpotent group.

By a theorem of Gromov [Gr], for a finitely generated group $G$ and for a field $k$, the Gelfand-Kirillov dimension of $k[G]$ (concerning the definition, see section 2) is finite if and only if of $G$ is almost nilpotent. So Theorem 1.2 implies the following corollary (see also Corollary 2.6).

Received June 23, 1998. 
COROLlary 1.3. Let $G$ be a finitely generated group, let $k$ be a finite field, and let $A$ be the group ring $k[G]$. Then the function $\zeta_{A}(s)$ converges if the Gelfand-Kirillov dimension of $A$ is finite.

Concerning the explicit computation of $\zeta_{A}(s)$ for group rings $A$, we have

THEOREM 1.4. Let $G$ be a finitely generated nilpotent group which has a central series

$$
G=G_{0} \supset G_{1} \supset \cdots \supset G_{r}=\{1\}
$$

such that

$$
G_{i} / G_{i+1} \cong Z \quad \text { for } 0 \leq i \leq r-1 .
$$

Let $R$ be a finitely generated commutative ring over $Z$, and let $A$ be the group ring $R[G]$. Then the function $\zeta_{A}(s)$ converges, and

$$
\zeta_{A}(s)=\zeta_{R^{\prime}}(s)
$$

where $R^{\prime}$ is the commutative ring $R\left[X_{1}, X_{1}^{-1}, \ldots, X_{r}, X_{r}^{-1}\right]$.

In section 2, we review the conjecture on the relationship between the convergence of our zeta functions and Gelfand-Kirilov dimensions of rings which is described in $[\mathrm{Fu}]$.

In section 3, we show the main line of the proof of Theorem 1.2, and give a part of the proof.

In section 4, we prove Theorem 1.4 and complete the proof of Theorem 1.2.

In section 5, we remark on the more strict form of our conjecture which is described in section 2 .

I would like to express my hearty gratitude to Professor Kazuya Kato who suggested me to study this subject, gave me a lot of essential advice, and encouraged me much.

Notes. In this paper, all rings are assumed to have a unit 1, and all ring homomorphisms are assumed to respect 1 . Fields are assumed to be commutative, though rings are not assumed to be commutative. For a ring $A$, an $A$-module means a left $A$-module on which 1 acts as the identity. As usual, $Z$ denotes the ring of integers. We sometimes call a representation of $A$, an $A$ representation.

\section{Review of the conjecture}

2.1. We introduce the definition of the Gelfand-Kirillov dimension. The Gelfand-Kirillov dimension is defined usually for algebras over fields. For a finitely generated algebra $A$ over a field $k$, the Gelfand-Kirillov dimension GK $\operatorname{dim}(A) \in\{t \in R ; t \geq 0\} \cup\{ \pm \infty\}$ of $A$ is defined as follows: Let $S$ be a finite 
set of the generators of $A$ over $k$. Then

$$
\mathrm{GK} \operatorname{dim}(A)=\limsup _{n \rightarrow \infty}\left(\frac{\log \operatorname{dim}_{k}\left(V_{n}(S)\right)}{\log n}\right)
$$

where $V_{n}(S)$ is the $k$-subspace $\sum_{\jmath=0}^{n} k S^{\jmath}$ of $A\left(S^{\jmath}=\left\{x_{1} \cdots x_{j} ; x_{1}, \ldots, x_{J} \in S\right\}\right)$. This is independent of the choice of $S$. If $A$ is commutative, $\mathrm{GK} \operatorname{dim}(A)$ coincides with the Krull dimension $\operatorname{dim}(A)$. (See [M-R] Chapter 8.)

For a commutative ring $R$ and a finitely generated $R$-algebra $A$, we define

$$
\mathrm{GK} \operatorname{dim}(A ; R)=\sup \left\{\mathrm{GK} \operatorname{dim}\left(A \otimes_{R} k(\mathfrak{p})\right)+\operatorname{dim}(R / \mathfrak{p}): \mathfrak{p} \in \operatorname{Spec}(R)\right\}
$$

where $k(\mathfrak{p})$ denotes the residue field of $\mathfrak{p}$. For a finitely generated $\operatorname{ring} A$ over $\boldsymbol{Z}$, we define the Gelfand-Kirillov dimension of $A$ by

$$
\mathrm{GK} \operatorname{dim}(A)=\mathrm{GK} \operatorname{dim}(A ; \boldsymbol{Z}) .
$$

GK $\operatorname{dim}(A)=\operatorname{dim}(A)$ holds again for a commutative finitely generated ring $A$ over $\boldsymbol{Z}$.

2.2. By Gromov [Gr], for a finitely generated group $G$ and for a field $k$, GK $\operatorname{dim}(k[G])$ is finite if and only if $G$ is almost nilpotent. From this we can deduce that for a finitely generated group $G$ and for a commutative finitely generated $\operatorname{ring} R \neq 0$ over $Z, G$ is almost nilpotent if and only if $\mathrm{GK} \operatorname{dim}(R[G])$ is finite. (To see this, we have to show that if $G$ is a finitely generated almost nilpotent group, then $\mathrm{GK} \operatorname{dim}\left(R[G] \otimes{ }_{z} k(\mathfrak{p})\right)$ is bounded by a number which is independent of $\mathfrak{p} \in \operatorname{Spec}(Z)$. This fact is a consequence of [Ba].) (We say the Gelfand-Kirillov dimension is finite also in the case it is $-\infty$.)

2.3. In $[\mathrm{Fu}]$, we observed that concerning the relationship between the convergence and the Gelfand-Kirillov dimension, the "modified Hasse zeta function" $\zeta_{A}^{*}(s)$ behaves better than $\zeta_{A}(s)$.

Let $A$ be a finitely generated ring over $Z$. We defined in [Fu], the modified Hasse zeta function $\zeta_{A}^{*}(s)$ of $A$ by

$$
\zeta_{A}^{*}(s)=\prod_{M}\left(1-N(M)^{-s}\right)^{-1}
$$

where $M$ runs over all isomorphism classes of simple $A$-modules such that $\sharp \operatorname{End}_{A}(M)$ is finite, and $N(M)=\sharp \operatorname{End}_{A}(M)$.

Remark that in this definition, $M$ itself need not be a finite module.

In $[\mathrm{Fu}]$, we formulated the following

CONJECTURE 2.4. Let $A$ be a finitely generated ring over $Z$. The function $\zeta_{A}^{*}(s)$ converges if and only if the Gelfand-Kirillov dimension of $A$ is finite.

The examples which we have computed by now satisfy the conjecture. Concerning Conjecture 2.4, we have the following proposition.

Proposition 2.5. Let $G$ be a finitely generated group having a polycyclic 
subgroup of finite index. Let $R$ be a commutative finitely generated ring over $Z$, and let $A$ be the group ring $R[G]$. Then

$$
\zeta_{A}(s)=\zeta_{A}^{*}(s)
$$

Especially, for $G$ and $A$ in Theorem 1.2, we have

$$
\zeta_{A}(s)=\zeta_{A}^{*}(s) \text {. }
$$

Proof. For $A=R[G]$ where $G$ is finitely generated group having a polycyclic subgroup of finite index, by [Ro], any simple $A$-module is finite.

Since an almost nilpotent group has a polycyclic subgroup of finite index, the latter part of Proposition 2.5 in obtained.

By Proposition 2.5 and by 2.2, Theorem 1.2 implies

COROLlARY 2.6. Let $A$ be a group ring of a finitely generated group over a commutative finitely generated ring. Then the function $\zeta_{A}^{*}(s)$ converges if the Gelfand-Kirillov dimension of $A$ is finite.

\section{Proof of the results}

We have the following propositions.

Proposition 3.1. Let $G$ be a finitely generated group, and let $H$ be a normal subgroup of $G$ of finite index. Let $R$ be a commutative finitely generated ring over $Z$. Let $A$ and $B$ be the group rings $R[G]$ and $R[H]$, respectively. If the function $\zeta_{B}(s)$ converges, then the function $\zeta_{A}(s)$ converges.

Proposition 3.2. Let $G$ be a finitely generated nilpotent group, let $R$ be a commutative finitely generated ring over $Z$, and let $A$ be the group ring $R[G]$. Then the function $\zeta_{A}(s)$ converges.

Theorem 1.2 is deduced easily from Propositions 3.1 and 3.2 as follows. Let $G, R$, and $A$ be as in Theorem 1.2.

Let $H^{\prime}$ be a nilpotent subgroup of $G$ of finite index, and let

$$
H=\bigcap_{g \in G} g H^{\prime} g^{-1}
$$

Then $H$ is a normal subgroup of $G$, and also of $H^{\prime}$ of finite index. Since a subgroup of a nilpotent group is nilpotent, $H$ is a nilpotent normal subgroup of $G$, and clearly of finite index. By well-known Lemma 3.3 below, $H$ is finitely generated. Let $B$ be the group ring $R[H]$. By Proposition 3.2, the function $\zeta_{B}(s)$ converges, and hence by Proposition 3.1 the function $\zeta_{A}(s)$ converges.

LEMMA 3.3. Let $G$ be a finitely generated group, and let $H$ be a subgroup of $G$ of finite index. Then $H$ is a finitely generated group. 
Proof. Let $S$ be a finite subset of $G$ which generates $G$, and let $T$ be a representative of $G / H$ in $G$ including 1 . For $g \in G$ and $t \in T$, write $g t=$ $t^{\prime} h_{g, t}\left(t^{\prime} \in T, h_{g, t} \in H\right)$. Then the finite set $\left\{h_{g, t} ; g \in S, t \in T\right\}$ generates $H$ as is easily seen.

In this section, we prove Proposition 3.1, and deduce Proposition 3.2 from Proposition 3.1 and Theorem 1.4. The proof of Theorem 1.4 is given in the next section.

LemMa 3.4. Let $G$ be a finitely generated nilpotent group. Then the group $G$ has a normal subgroup $G^{\prime}$ of finite index which has a central series

$$
G^{\prime}=G_{0}^{\prime} \supset G_{1}^{\prime} \supset \cdots \supset G_{r}^{\prime}=\{1\}
$$

such that

$$
G_{i}^{\prime} / G_{i+1}^{\prime} \cong Z \text { for } 0 \leq i \leq r-1
$$

Proof. We prove this by induction on $n=\sum_{l=0}^{r-1} \operatorname{rank}\left(G_{i} / G_{i+1}\right)$ where $\left\{G_{i}\right\}_{l}$ is the lower central series of $G . \quad\left(G_{i} / G_{i+1}(0 \leq i \leq r-1)\right.$ are finitely generated by [Se] Corollary 7.) We may assume $n \geq 1$. Then the center of $G$ is infinite by [Se], Section 1, Corollary 6. Take an infinite cyclic subgroup $H$ of the center of $G$. By induction, $G / H$ has a normal subgroup $G^{\prime \prime}$ of finite index having a central series as in Lemma 3.4. Let $G^{\prime}$ be the inverse image of $G^{\prime \prime}$ in $G$. Then $G^{\prime}$ has a central series as in Lemma 3.4.

Proposition 3.2 follows from Theorem 1.4 and Lemma 3.4 by considering Proposition 3.1.

We prove Proposition 3.1 in 3.5-3.7.

Let $f_{S}(A)$ and $f_{S}(B)$ be the set of all isomorphism classes of finite simple $A$ modules, and all isomorphism classes of finite simple $B$-modules, respectively.

3.5. For a simple $B$-module $N$ (resp. $A$-module $M$ ), we denote by $[N]$ (resp. $[M]$ ) the element of $f s(B)$ (resp. $f s(A)$ ) which is represented by $N$ (resp. $M$ ). Let $G \backslash f s(B)$ be the quotient of $f s(B)$ by the equivalence that $\left[N_{1}\right],\left[N_{2}\right] \in f_{s}(B)$ are equivalent if and only if $N_{2} \cong \sigma N_{1}$ for some $\sigma \in G$ as $B$-modules. Here $\sigma N=$ $\{\sigma x ; x \in N\}$ is the set of symbols $\sigma x(x \in N)$ which is regarded as a $B$-module in the natural way. (For $b \in B$ and $x \in N, b \sigma x$ is defined to be $\sigma\left(\sigma^{-1} b \sigma\right) x$.)

We consider the diagram

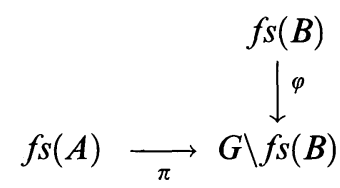

where $\varphi$ is the canonical map and $\pi$ is the following map. Let $M$ be a simple $A$-module. Let $N$ be a simple $B$-submodule of $M$. We define $\pi([M])=[N]$ $\bmod G$. We show that this map $\pi$ is well-defined. Since the subgroup $\sum_{\tau \in G} \tau N$ 
of $M$ is stable under the action of $A$, it coincides with $M$. Hence any simple $B$-submodule of $M$ is isomorphic to $\tau N$ for some $\tau \in G$. So the map $\pi$ is well-defined.

LEMMA 3.6. Let $l=[G: H]$.

(1) For any $[N] \in f s(B)$, we have $\sharp \pi^{-1}(\varphi([N])) \leq l$.

(2) For any simple $A$-module $M$ and for any simple $B$-module $N$ such that $\varphi([N])=\pi([M])$, we have $N(N) \leq N(M)^{1 / l}$.

As the function $\zeta_{B}(s)$ converges, Proposition 3.1 follows from Lemma 3.6. So we prove Lemma 3.6 in 3.7.

3.7. (1) Since any finite simple $A$-module $M$ such that $\pi([M])=[N]$ is isomorphic to some subquotient $A$-module of $A \otimes_{B} N, \sharp \pi^{-1}(\varphi([N])) \leq$ length $_{A}\left(A \otimes_{B} N\right) \leq$ length $_{B}\left(A \otimes_{B} N\right)=l$.

(2) Let $\mathrm{m}_{N}$ be the annihilator of $N$. Let $F$ be the center of $B / \mathrm{m}_{N}$, that is, $\sharp F=N(N)$. Let $G_{N}=\left\{g \in G ; g \mathfrak{m}_{N} g^{-1}=\mathfrak{m}_{N}\right\}$. Let $I_{N}=\{g \in G$; the action of $g() g^{-1}$ on $F$ is identity $\}$. It holds that $G \supset G_{N} \supset I_{N} \supset H$, and $I_{N}$ is a normal subgroup of $G_{N}$. An element of $G_{N} / I_{N}$ can be considered as an element of the automorphism of $F$. Let $F_{0}$ be the subfield of $F$ which is stabilized by $G_{N} / I_{N}$. Then $\left[F: F_{0}\right]=\sharp\left(G_{N} / I_{N}\right) \leq l$.

We show that $F_{0}$ is embedded in $\operatorname{End}_{A}(M)$ as a subfield. Then we obtain that $\sharp \operatorname{End}_{A}(M)=N(M) \geq N(N)^{1 / l}$. It holds

$$
M \cong \bigoplus_{i=0}^{n-1} \tau_{i} N^{\prime} \quad \text { as } B \text {-modules }
$$

where $\left\{\tau_{0}, \ldots, \tau_{n-1}\right\}$ be the system of the representatives of $G / G_{N}$, and $N^{\prime}=\left\{x \in M ; a_{x}=0\right.$ for any $\left.a \in \mathfrak{m}_{N}\right\}$. For $\alpha \in F_{0}$, we define

$$
\alpha: \bigoplus_{l=0}^{n-1} \tau_{i} N^{\prime} \rightarrow \bigoplus_{l=0}^{n-1} \tau_{i} N^{\prime} ; \quad \bigoplus_{l=0}^{n-1} x_{l} \mapsto \bigoplus_{l=0}^{n-1}\left(\tau_{i} \alpha \tau_{l}^{-1} x_{l}\right) .
$$

We prove that this map $\alpha \in \operatorname{End}_{A}(M)$. It is sufficient to prove that for $\tau \in G$ such that $\tau \tau_{i} N^{\prime}=\tau_{g(i)} N^{\prime}(0 \leq i \leq n-1,0 \leq g(i) \leq n-1), \tau \tau_{i} \alpha \tau_{l}^{-1}=\tau_{g(i)} \alpha \tau_{g(i)}^{-1} \tau$ on $\tau_{i} N^{\prime}$. That is, $\left(\tau_{g(i)}^{-1} \tau \tau_{l}\right) \alpha\left(\tau_{g(i)}^{-1} \tau \tau_{l}\right)^{-1}=\alpha$ on $N^{\prime}$, since

$$
\tau_{g(i)}^{-1} \tau \tau_{l}: N^{\prime} \rightarrow \tau_{i} N^{\prime} \rightarrow \tau_{g(i)} N^{\prime} \rightarrow N^{\prime}
$$

As $\tau_{g(i)}^{-1} \tau \tau_{l} \in G_{N}$, by the definition of $\alpha$, the above equations holds. Hence $F_{0} \subset \operatorname{End}_{\mathrm{A}}(M)$.

Hence we obtain the result of Proposition 3.1.

\section{Proof of the results. 2}

4.1. In this section, we prove Theorem 1.4 by using Proposition 4.2 below. First, we prepare to describe Proposition 4.2. Let $A$ be a finitely generated ring 
over $\boldsymbol{Z}$. The Hasse zeta function $\zeta_{A}(s)$ has the expression

$$
\zeta_{A}(s)=\prod_{r \geq 1} \zeta_{A, r}(s)
$$

where $r$ runs over integers $\geq 1$ and,

$$
\zeta_{A, r}(s)=\prod_{p} \exp \sum_{n=1}^{\infty} \frac{\sharp \mathcal{S}_{A, r}\left(\boldsymbol{F}_{p^{n}}\right)}{n}\left(p^{-s}\right)^{n}
$$

where $\mathfrak{S}_{A, r}$ is a certain scheme of finite type over $Z, p$ runs over prime numbers, and $\boldsymbol{F}_{p^{n}}$ is a finite field with $p^{n}$ elements, so the function $\zeta_{A, r}(s)$ coincides with the product of Weil's zeta functions of $\mathfrak{S}_{A, r} \otimes_{Z} \boldsymbol{F}_{p}$ [We] for all prime numbers $p$. We do not review the definition of $\mathfrak{\Xi}_{A, r}$, but what we need in this paper is that for the algebraic closure $K$ of $\boldsymbol{F}_{p}, \mathfrak{S}_{A, r}(K)$ is identified with the set of all isomorphism classes of $r$-dimensional irreducible representations of $A$ over $K$, and $\mathfrak{S}_{A, r}\left(\boldsymbol{F}_{p^{n}}\right)$ is identified with the $\operatorname{Gal}\left(K / \boldsymbol{F}_{p^{n}}\right)$-fixed part of $\mathfrak{S}_{A, r}(K)$.

Let $G, R$ and $A$ be as in Theorem 1.4. We may assume that $R$ is a finite field of characteristic $p>0$, for $\zeta_{R}(s), \zeta_{A}(s)$ are products of $\zeta_{R / \mathfrak{m}}(s), \zeta_{A / \mathfrak{m} A}(s)$ over all maximal ideals $\mathrm{m}$ of $R$, respectively. So assume $R$ is a finite field $k$ of characteristic $p$, and let $K$ be the algebraic closure of $k$.

Let $\mathfrak{\Xi}_{A}=\bigsqcup_{r \geq 1} \mathfrak{\Xi}_{A, r}$, and for an extension $k^{\prime}$ of $k$, let $\mathfrak{\Xi}_{A}^{k}\left(k^{\prime}\right)$ be the set of $k^{\prime}$-rational points of $\mathfrak{S}_{A}$ as a $k$-scheme.

For a finite dimensional irreducible representation $N$ of $B$ (resp. $M$ of $A$ ) over $K$, we denote by $[N]$ (resp. $[M])$ the element of $\mathfrak{\Xi}_{B}^{k}(K)\left(\right.$ resp. $\left.\mathfrak{S}_{A}^{k}(K)\right)$ which is represented by $N$ (resp. $M$ ).

We have

Proposition 4.2. Let $G, k, K$, and $A$ be as above $(A=k[G])$. Let $\boldsymbol{F}_{q}$ be a finite extension of $k$ in $K$ which has $q$ elements, and let

$$
\text { Frob }_{q}: K \rightarrow K ; x \mapsto x^{q} .
$$

Let $\theta: G \rightarrow G$ be an automorphism of $G$ such that $\theta\left(G_{i}\right)=G_{i}$ for $0 \leq i \leq r$ and $\theta: G_{i} / G_{i+1} \rightarrow G_{i} / G_{i+1}$ is the identity map of $G_{i} / G_{i+1}$ for $0 \leq i \leq r-1$. Extend the action of $\theta$ on $G$ to $A$ where the action of $\theta$ on $k$ is trivial. Then $\sharp\{[M] \in$ $\left.\mathfrak{S}_{A}^{k}(K) ; \operatorname{Frob}_{q}([M])=\theta([M])\right\}=(q-1)^{r}$, a number which does not depend on the automorphism $\theta$.

Now, we prove Theorem 1.4 by using Proposition 4.2.

It is sufficient to prove that for any finite extension $\boldsymbol{F}_{q}$ of $k$ in $K$, $\sharp \Im_{A}^{k}\left(\boldsymbol{F}_{q}\right)=(q-1)^{r}$. By taking the identity map of $G$ as $\theta$ in Proposition 4.2, we obtain Theorem 1.4 .

We prove Proposition 4.2 by induction on $r$. The outline of the proof is as follows. Assume $r \geq 1$, and let $B=k\left[G_{1}\right]$. Fix an element $\alpha$ of $G$ whose image in $G / G_{1} \cong Z$ is a generator, let $f: G_{1} \rightarrow G_{1}$ be the automorphism of $G_{1}$ defined by $x \mapsto \alpha x \alpha^{-1}$, and extend $f$ to an automorphism of $B$ in the natural way. Let 
$f \backslash \Im_{B}^{k}(K)$ be the quotient of $\Im_{B}^{k}(K)$ by the action of $f$. As we will show later, in the category of $\operatorname{Gal}(K / k)$-sets, $\Im_{A}^{k}(K)$ is a $K^{*}$-principal homogeneous space over $f \backslash \Im_{B}^{k}(K)$. The action of $\theta: \Im_{B}^{k}(K) \rightarrow \Im_{B}^{k}(K)$ commutes with $f$ and hence induces $f \backslash \Im_{B}^{k}(K) \rightarrow f \backslash \Im_{B}^{k}(K)$. From Lemma 4.3 below by using our induction, we will deduce

$$
\sharp\left\{x \in f \backslash \Im_{B}^{k}(K) ; \operatorname{Frob}_{q}(x)=\theta(x)\right\}=(q-1)^{r-1} .
$$

The key point will be the fact $\sharp\left\{x \in \Xi_{B}^{k}(K)\right.$; $\left.\operatorname{Frob}_{q}(x)=\theta f^{l}(x)\right\}=(q-1)^{r-1}$ for all $i$ by our induction which is applied to the automorphisms $\theta f^{l}$ of $G_{1}(i \in Z)$. From the fact $\mathfrak{S}_{A}^{k}(K)$ is a $K^{*}$-principal homogeneous space over $f \backslash \mathfrak{S}_{B}^{k}(K)$, we will deduce

$$
\begin{aligned}
\sharp\{x \in & \left.\mathfrak{S}_{A}^{k}(K) ; \operatorname{Frob}_{q}(x)=\theta(x)\right\} \\
& =\sharp\left\{x \in f \backslash \mathfrak{\Im}_{B}^{k}(K) ; \operatorname{Frob}_{q}=\theta(x)\right\} \cdot \sharp\left\{x \in K^{*} ; x^{q}=x\right\} \\
& =(q-1)^{r-1} \cdot(q-1)=(q-1)^{r} .
\end{aligned}
$$

The following lemma will be applied to the proof of Proposition 4.2 by taking $Y=\mathfrak{S}_{B}^{k}(K), F=\theta^{-1}$ Frob $_{q}$.

Lemma 4.3. Let $Y$ be a set, and let $f: Y \rightarrow Y$ be a bijection. Let $F: Y \rightarrow$ $Y$ be a map which commutes with $f$. Assume that for any $i \in Z,\{y \in Y$; $\left.F(y)=f^{l}(y)\right\}$ is a finite set, and its order does not depend on $i$. Then

$$
\sharp\{y \in Y ; F(y)=y\}=\sharp\{x \in f \backslash Y ; F(x)=x\} .
$$

Proof. For each $i \in Z$, put $F_{l}=\left\{y \in Y ; F(y)=f^{\prime}(y)\right\}$. Let $\varphi: Y \rightarrow f \backslash Y$ be the canonical map. For $y \in F_{i}$, as $\varphi^{-1}(\varphi(y)) \subset F_{i}$ (since $F$ commutes with $f$ ) and as $F_{i}$ is a finite set, $\sharp \varphi^{-1}(\varphi(y))=l(y)$ is finite. Then $y \in F_{l+l(y) m}$ for all $m \in \boldsymbol{Z}$ and $y$ is not contained in $F_{j}$ if $j \neq i+l(y) m$ for any $m \in Z$. Hence there exists $n \in Z, n \geq 1$ such that $F_{i+n}=F_{i}$ for all $i \in Z$. So for the canonical map $\phi: \coprod_{0 \leq l<n} F_{i} \rightarrow \mathfrak{F}$ where $\mathfrak{F}=\{x \in f \backslash Y ; F(x)=x\}$, the set $\phi^{-1}(\phi(x))$ is of order $n$ for any $x \in \mathfrak{F}$. Hence $\sharp \mathfrak{F}=\sharp F_{i}=\sharp F_{0}=\sharp\{y \in Y ; F(y)=y\}$, a finite number which does not depend on $i \in \boldsymbol{Z}$.

We prove Proposition 4.2.

We prove by induction on $r$. Assume $r \geq 1$, and let $B$ be the group ring $k\left[G_{1}\right]$ where $G_{1}$ is the subgroup of $G$ in Theorem 1.4. In the exact sequence

$$
1 \rightarrow G_{1} \rightarrow G \rightarrow Z \rightarrow 1
$$

let $f: G_{1} \rightarrow G_{1}$ be the automorphism of $G_{1}$ defined by $x \mapsto \alpha x \alpha^{-1}$ where $\alpha$ is an element of $G$ whose image in $Z$ is 1 . The ring $A$ can be expressed as the following skew Laurent polynomial ring: $\left\{\sum_{l=-N}^{N} a_{i} T^{i} ; N \geq 0, a_{i} \in B\right\}$ in which $T$ is an indeterminate and the multiplication is defined by the rule $T a=f(a) T$ 
$(a \in B)$. When we consider the $\operatorname{ring} A$ as the above skew Laurent polynomial ring, we can identify the element $\alpha$ of $A$ as $T$.

We define a surjective map $\pi: \Im_{A}^{k}(K) \rightarrow f \backslash \Im_{B}^{k}(K)$.

Let $M$ be a finite dimensional irreducible representation of $A$ over $K$, and let $N$ be a $B$-subrepresentation of $M$. We define $\pi([M])=[N] \bmod f$. We show that this map $\pi$ is well-defined. Since the subgroup $\sum_{l \in Z} T^{\imath} N$ of $M$ is stable under the action of $A$, it coincides with $M$. Here $T^{\imath} N=\left\{T^{\imath} x ; x \in N\right\}(i \in Z)$ is the set of symbols $T^{l} x(x \in N)$ which is regarded as a $B$-representation in the natural way. (For $b \in B$ and $x \in N, T^{l} x$ is defined to be $T^{l} f^{-1}(b) x$.) Hence any simple $B$-submodule of $M$ is isomorphic to $T^{l} N$ for some $i \in Z$ and $T^{l} N \cong$ $f^{-l}(N)$.

We assume that $\sharp\left\{[N] \in \Im_{B}^{k}(K) ; \operatorname{Frob}_{q}([N])=\theta([N])\right\}=(q-1)^{r-1}$ as the inductive hypothesis, then we will prove that $\sharp\left\{[M] \in \mathfrak{S}_{A}^{k}(K)\right.$; $\operatorname{Frob}_{q}([M])=$ $\theta([M])\}=(q-1)^{r}$.

Lemma 4.4. Let $N$ be a finite dimensional irreducible representation of $B$ over $K$, and let $n$ be the minimal integer $>0$ such that $f^{n}([N])=[N]$ in $\mathfrak{S}_{B}^{k}(K)$. Let $J_{N} \subset B_{K}=B \otimes_{k} K$ be the annihilator of $N$, and let

$$
D=\left\{g \in\left(B_{K} / J_{N}\right)^{*} ; g\left(b \bmod J_{N}\right) g^{-1}=f^{n}(b) \bmod J_{N} \text { for all } b \in B_{k}\right\} .
$$

On the other hand, let $C$ be the set of isomorphism classes of finite dimensional irreducible representation $M$ of $A$ over $K$ such that $\pi([M])=[N] \bmod f$. Then there exists a bijection between $C$ and $D$ which preserves the action of $\operatorname{Gal}(K / k)$.

(Note that $f^{n}\left(J_{N}\right)=J_{N}$. Since $B_{K} / J_{N}$ is isomorphic over $K$ to $M_{r}(K)$ for some $r \geq 1$, by the theorem of Skolem-Noether, the automorphism of $B_{K} / J_{N}$ induced by $f^{n}$ coincides with $g() g^{-1}$ for some $g \in\left(B_{K} / J_{N}\right)^{*}$.)

Since $D$ is a $K^{*}$-principal homogeneous space (by the multiplication in $\left.\left(B_{K} / J_{n}\right)^{*}\right)$, Lemma 4.4 implies that $C$ has a structure of a $K^{*}$-principal homogeneous space, and then $\mathfrak{S}_{A}^{k}(K)$ has a structure of a $K^{*}$-principal homogeneous space over $f \backslash \mathfrak{\Im}_{B}^{k}(K)$.

Proof. We define the map $D \rightarrow C$ as $g \mapsto \bigoplus_{i=0}^{n-1} T^{\imath} N$ for $g \in N$, where the $A$-module structure on $\bigoplus_{i=0}^{n-1} T^{i} N$ is as follows. The action of $B$ is the natural one and the action of $T$ is given by

$$
\sum_{l=0}^{n-1} T^{l} x_{l} \mapsto \sum_{l=0}^{n-2} T^{l+1} x_{l}+g x_{n-1}
$$

It is easy to see that this map $D \rightarrow C$ is injective. We prove that it is surjective. Let $[M] \in \mathfrak{S}_{A}^{k}(K)$, and let $[N] \in \mathfrak{S}_{B}^{k}(K)$ be an isomorphism class of an irreducible subrepresentation of $B$ in $M$. We prove $M=\bigoplus_{i=0}^{n-1} T^{l} N$. Let $N^{\prime}=$ $\left\{x \in M ; a x=0\right.$ for any $\left.a \in J_{N}\right\}$. For any $g_{1} \in D$, the action of $g_{1}^{-1} T^{n}\left(\bmod J_{N}\right)$ commutes with the action of $B$ on $N^{\prime}$. Assume $N^{\prime}$ as a $B[s]$-module where $s$ is 
an indeterminant and the action of $s$ is given by $g_{1}^{-1} T^{n}\left(\bmod J_{N}\right)$. Let $N^{\prime \prime}$ be an irreducible $B[s]$-subrepresentation of $N^{\prime}$. Since the action of $s$ commutes with the action of $B[s]$, the action of $s$ on $N^{\prime \prime}$ is a scalar. So $N^{\prime \prime}$ is irreducible also of $B$. Hence $N^{\prime \prime} \cong N$ as $B$-representations. As $\sum_{l=0}^{n-1} T^{l} N^{\prime \prime}=\bigoplus_{i=0}^{n-1} T^{l} N^{\prime \prime}$ is stable under the action of $T$, it coincides with $M$. We also obtain that $M=\bigoplus_{i=0}^{n-1} N$. The action of $T^{n}$ in $M$ is regarded as an element of $\left(B_{K} / J_{N}\right)^{*}$, and it is the element of $D$ which corresponds to $M$ by the map $D \rightarrow C$. So the map $D \rightarrow C$ is surjective. Hence we obtain that there exists a bijection between $C$ and $D$ which preserves the action of $\operatorname{Gal}(K / k)$.

As $\mathfrak{S}_{A}^{k}(K)$ has a structure of a $K^{*}$-principal homogeneous space over $f \backslash \mathfrak{S}_{B}^{k}(K)$, for $[N] \bmod f \in f \backslash \mathfrak{S}_{B}^{k}(K)$ such that $\operatorname{Frob}_{q}([N] \bmod f)=\theta([N] \bmod f)$,

$$
\begin{aligned}
\sharp\{[M] & \left.\in \mathbb{S}_{A}^{k}(K) ; \operatorname{Frob}_{q}([M])=\theta([M]), \pi([M])=[N] \bmod f\right\} \\
= & \sharp\left\{a \in K^{*} ; \operatorname{Frob}_{q}(a[M])=\theta(a[M])\right\} \\
= & \sharp\left\{a \in K^{*} ; a^{q} \operatorname{Frob}_{q}([M])=a \theta([M])\right\} \\
= & \sharp\left\{a \in K^{*} ; a^{q-1} \operatorname{Frob}_{q}([M])=\theta([M])\right\} .
\end{aligned}
$$

For $b \in K^{*}$ such that $\theta([M])=b \operatorname{Frob}_{q}([M])$, the above order is the same as the number of the solutions $a$ of the equation $a^{q-1}=b$, that is, $q-1$.

Hence

$$
\begin{aligned}
& \sharp\left\{[M] \in \mathfrak{S}_{A}^{k}(K) ; \operatorname{Frob}_{q}([M])=\theta([M])\right\} \\
& =(q-1) \cdot \sharp\left\{[N] \bmod f \in f \backslash \mathfrak{\Im}_{B}^{k}(K) ; \operatorname{Frob}_{q}([N] \bmod f)\right. \\
& =\theta([N] \bmod f)\} .
\end{aligned}
$$

We prove that $\sharp\left\{[N] \bmod f \in f \backslash \Im_{B}^{k}(K) ; \operatorname{Frob}_{q}([N] \bmod f)=\theta([N] \bmod f)\right\}=$ $(q-1)^{r-1}$. We use Lemma 4.3 by taking $Y=\Xi_{B}^{k}(K), F=\theta^{-1}$ Frob $_{q}$. We show that we can apply Lemma 4.3. Let $\rho_{N}$ be a $K$-homomorphism from $B_{K}$ into $M_{r}(K)$ for some $r \geq 1$ which represents the action of $B$ in $N$. Since it is clear that $\operatorname{Frob}_{q}$ commutes with $f$, we show that $\theta$ commutes with $f$. We have

$$
\begin{aligned}
\rho_{\theta^{-1} f^{-1} \theta f(N)}(b) & =\rho_{N}\left(f^{-1} \theta^{-1} f \theta(b)\right)=\rho_{N}\left(\alpha^{-1}\left(\theta^{-1}\left(\alpha \theta(b) \alpha^{-1}\right)\right) \alpha\right) \\
& =\rho_{N}\left(\alpha^{-1} \theta^{-1}(\alpha) b \theta^{-1}(\alpha)^{-1} \alpha\right)=\rho_{N}\left(u b u^{-1}\right)
\end{aligned}
$$

where $u=\alpha^{-1} \theta^{-1}(\alpha)$, and $u \in G_{1} \subset B$ as it is a property of $\theta$. Hence $F$ commutes with $f$.

Take $\theta f^{i}$ as $\theta$ of Proposition 4.2. From our hypothesis of the induction, $\left\{[N] \in \mathfrak{S}_{B}^{k}(K) ; F([N])=f^{\imath}([N])\right\}$ is a finite set, and its order $(q-1)^{r-1}$ does not depend on $i$. So we can apply Lemma 4.3. Hence 


$$
\begin{aligned}
& \sharp\left\{[N] \bmod f \in f \backslash \Im_{B}^{k}(K) ; \operatorname{Frob}_{q}([N] \bmod f)=\theta([N] \bmod f)\right\} \\
& \quad=\sharp\left\{[N]^{\cdot} \in \Im_{B}^{k}(K) ; \operatorname{Frob}_{q}([N])=\theta([N])\right\} \\
& =(q-1)^{r-1} .
\end{aligned}
$$

Hence we accomplish the proof of Proposition 4.2.

\section{Remark}

In [Fu], we made a more strict conjecture on the relationship between the convergence of the zeta function and the Gelfand-Kirillov dimension of its ring.

CONJECTURE 5.1. Let $R$ be a commutative finitely generated ring over $Z$, and let $A$ be a finitely generated R-algebra. We define the "zeta dimension" $\zeta \operatorname{dim}(A) \in\{t \in \boldsymbol{R} ; t \geq 0\} \cup\{ \pm \infty\}$ of $A$ by

$$
\zeta \operatorname{dim}(A)=\operatorname{Inf}\left\{d \in \boldsymbol{R} ; \zeta_{A}^{*}(s) \text { converges if } \operatorname{Re}(s)>d\right\} .
$$

Then

$$
\zeta \operatorname{dim}(A) \leq \mathrm{GK} \operatorname{dim}(A ; R) .
$$

5.2. For a finitely generated almost nilpotent group $G$, and for the group ring $A=R[G]$, if we take a nilpotent subgroup of $G$ of finite index sufficiently small, then we may assume that $G_{0}$ is a normal subgroup of $G$. Let

$$
G \supset G_{0} \supset G_{1} \supset \cdots \supset G_{r}=\{1\}
$$

be a lower sequence of subgroups of $G$ where

$$
G_{0} \supset G_{1} \supset \cdots \supset G_{r}=\{1\}
$$

is the lower central series of $G_{0}$. Then we conjecture that

$$
\zeta \operatorname{dim}(A)=\operatorname{dim}(R)+\sum_{i=0}^{r-1} \operatorname{rank}_{Z}\left(G_{i} / G_{i+1}\right) .
$$

By [Ba], 5.2 is compatible with 5.1 .

In the case $G$ is the group of Theorem 1.4, by Theorem 1.4, the conjecture in 5.2 is satisfied.

5.3. Let $B \rightarrow A$ be a homomorphism of finitely generated $R$-algebras, and assume that $A$ is of finite type as a $B$-module. We conjecture that

$$
\zeta \operatorname{dim}(A)=\zeta \operatorname{dim}(B) .
$$

From this conjecture and Theorem 1.4, we conjectured 5.2.

But it seems difficult to solve this problem. 


\section{REFERENCES}

[Ba] H. BAss, The degree of polynomial growth of finitely generated nilpotent groups, Proc. London Math. Soc. (3), 25 (1972), pp. 603-614.

[Ro] J. E. Rosenblade, Group rings of polycyclic groups, J. Pure Appl. Algebra, 3 (1993), pp. 307-328.

[Se] D. Segal, Polycyclic Groups, Cambridge Tracts in Math., 82, Cambridge Unıversity Press, 1983.

[S-F] H. Strade And R. Farnsteiner, Modular Lie Algebras and their Representations, Monogr. Textbooks Pure Appl. Math., 116, Marcel Dekker, New York, 1988.

[We] A. WeIL, Numbers of solutions of equations over finite fields, Bull. Amer. Math. Soc., 55 (1949), pp. 497-508.

[Fu] T. FuKaYA, Hasse zeta functions of non-commutative rings, J. Algebra, 208 (1998), pp. 304-342.

Graduate School of Mathematical Sciences

THE UNIVERSITY OF TOKYO

3-8-1 Komaba Meguro-KU

TOKYO, 153-8914

JAPAN 